\title{
Diagnóstico e tratamento de úlcera hipertensiva de Martorell: artigo de revisão
}

\begin{abstract}
RESUMO | Introdução: A úlcera hipertensiva de Martorell é uma ferida crônica, associada a hipertensão arterial sistêmica, de difícil manejo clínico e subdiagnosticada. Objetivo: Investigar os métodos de diagnóstico e os tratamentos existentes para a UHM. Método: Estudo descritivo e qualitativo de revisão integrativa com a análise sistemática de referências bibliográficas nas bases de dados PubMed, SciELO, MEDLINE e LILACS no período de 2016 e 2021, e periódicos da CAPES. Resultados: Clinicamente o paciente apresenta uma ferida pequena, de forma arredondada, superficial, com margens necróticas e cianóticas e eritema perilesional eventual. Os pulsos distais usualmente estão presentes e há ausência de edema e varizes locais. De todos os sinais e sintomas relatados, destaca-se a dor, descrita como intensa e desproporcional à sua dimensão. Conclusão: O cerne ao diagnóstico é avaliação clínica e o tratamento é a normalização da pressão arterial sistêmica e curativos regulares e, uso de analgésicos, antibióticos e vasodilatadores periféricos.
\end{abstract}

Descritores: Ferimentos e Lesões; Úlcera Cutânea; Hipertensão; Úlcera de perna.

\begin{abstract}
I Introduction: Martorell's hypertensive ulcer is a chronic wound, associated with systemic hypertension with difficult clinical management and it is often underdiagnosed. Goal: To investigate the diagnostic method and current treatments for Martorell's hypertensive ulcer disease. Method: A descriptive and qualitative study of integrative literature review with a systematic analysis of bibliographic references from PubMed, SciELO, MEDLINE and LILACS databases, ranging from 2016 to 2021, as well as selected articles from CAPES journals. Results: patients usually present with a small, rounded and superficial wound with cyanotic and necrotic edges and occasional perilesional erythema. Distal pulses are usually present and there is an absence of edema and local varicose veins. From all the signs and symptoms reported by patients with this type of injury, it is possible to point out that the pain is intense and disproportionate to its dimension. Conclusion: The core of diagnosis is the clinical evaluation and the treatment is controlled systemic blood pressure, regular bandages and, if needed, analgesics, antibiotics and peripheral vasodilators.
\end{abstract}

Keywords: Wounds and Injuries; Cutaneous Ulcer; Hypertension; Leg Ulcer

RESUMEN | Introdución: La úlcera hipertensiva de Martorell es una herida crónica, asociada a la hipertensión arterial sistémica, de difícil manejo clínico y muchas veces subdiagnosticada. Objetivo: Investigar los métodos de diagnóstico y tratamientos existentes para este tipo de lesión. Método: Se realizó un análisis sistemático de referencias bibliográficas en las bases de datos PubMed, SciELO, MEDLINE y LILACS en el período de publicación entre 2016 y 2021, además de artículos seleccionados de revistas CAPES. Resultados: Clínicamente el paciente suele presentarse con una pequeña herida, redondeada, superficial, con márgenes necróticos y cianóticos con eritema perilesional ocasional. Los pulsos distales suelen estar presentes y hay ausencia de edema y venas varicosas locales. De todos los signos y síntomas reportados por los pacientes con este tipo de lesiones, se destaca el dolor, que se describe como intenso y desproporcionado con la zona de la herida y su tamaño. Conclusión: La base del tratamiento es la normalización de la presión arterial sistémica y los apósitos regulares y, si necesario, analgésicos, antibióticos y vaso dilatadores periféricos.

Palabras claves: Heridas y Traumatismos; Úlcera de piel; Hipertensión; úlcera de pierna

\section{Hélio Martins do Nascimento Filho}

Enfermeiro. Mestre em Ciência, Tecnologia e Gestão Aplicadas à Regeneração Tecidual pela Unifesp. Enfermeiro na Unidade Saúde da Família / USF em Conselheiro Lafaiete - MG. Professor na Pós-graduação em Enfermagem Dermatológica com Ênfase no Tratamento de Feridas na Faculdade FAIPE (Cuiabá-MT).

ORCID-ID: 0000-0003-3700-3910

\section{Fabíola Arantes Ferreira}

Enfermeira. Mestre em Enfermagem em Terapia Intensiva, possui graduação em Enfermagem pela UEMG.

ORCID: 0000-0001-7360-820X.

\section{Elisângela Soares da Silva Reis}

Enfermeira. Especialista em Enfermagem de Média Alta Complexidade - Estomaterapia pela UFMG e em Enfermagem em Urgência Emergência e Trauma pela PUC de Minas Gerais (2011). Atualmente é Enfermeira da Hospital Sofia Feldman.

ORCID: 0000-0001-9753-062X.

\section{Daniela Tinti Moreira Borges}

Enfermeira. Mestre Profissional em Ciências pela UNIFESP/SP; Estomaterapeuta, pela FAMERP/SP (2019). Docente de Graduação em Enfermagem na AEMS (Faculdades Integradas de Três Lagoas/MS) ORCID: 0000-0001-8890-8918.
Flávia Carla Takaki Cavichioli

Enfermeira. Mestre em Ciência, Tecnologia e Gestão Aplicadas a Regeneração Tecidual pela UNIFESP.

ORCID-ID: 0000-0003-2277-9548.

\section{Lara Mendes Chaer Rezende Costa}

Médica Especialista em Cirurgia Geral e em Angiologia e Cirurgia Vascular, possui graduação em: Medicina pela Faculdade de Ciências Médicas e da Saúde de Juiz de Fora.

ORCID: 0000-0001-7131-0960.

/Recebido em: 21/07/2021

Aprovado em: 28/09/2021 
INTRODUÇÃO

$\mathrm{F}$ erida ou lesão cutânea pode ser definida como o rompimento de uma ou mais camadas da pele humana com alterações da anatomia e da fisiologia do tegumento que leva a falhas no processo de regeneração tecidual e pode tornar-se uma condição crônica e neste caso descrita como problema de saúde pública no Brasil e no mundo com alto custos de tratamento, impactos sociais e emocionais $^{(1)}$.

As úlceras de pernas são responsáveis pela grande maioria das lesões cutâneas e são mais prevalentes em mulheres. Doenças como Diabetes Mellitus (DM), Hipertensão Arterial Sistêmica (HAS), Doença Arterial Obstrutiva Periférica (DAOP), dislipidemias, anemias e outros fatores como idade, gênero, tabagismo, etilismo e sobrepeso, pode favorecer o seu desenvolvimento $^{(1,2,4,5)}$.

Nos Estados Unidos da América (EUA), a incidência de pacientes com feridas complexas varia entre 1 e $4 \%$ e nos idosos essa taxa chega a $6 \%$ dos americanos. Feridas possuem etiologias distintas, com destaque para as chamadas úlceras vasculogênicas que são responsáveis por cerca de $80 \%$ do total de casos, com destaque para a úlceras de etiologias venosa (até 90\%), úlcera arterial (de 5 a 25\%), úlcera mista (de 3 a $8 \%$ ) e aquelas relacionadas à vasculite (de 1 a $3 \%)^{(2,3,6)}$.

A Úlcera hipertensiva de Martorell (UHM) está relacionada a isquemia de arteríolas decorrente de hipertensão arterial sistêmica sem controle adequado por longos períodos e foi descrita pela primeira vez em 1945, pelo médico cardiologista Otzet Fernando Martorell, em Barcelona, na Espanha. Martorell identificou a lesão em quatro mulheres obesas e hipertensas ${ }^{(7-9)}$.

A apresentação clínica da UHM são lesões solitárias que muitas vezes estão simetricamente presentes nas extremidades e face externa dos membros infe-
As úlceras de pernas são responsáveis pela grande maioria das lesões cutâneas e são mais prevalentes em mulheres. Doenças como Diabetes Mellitus (DM), Hipertensão Arterial Sistêmica (HAS), Doença Arterial Obstrutiva Periférica (DAOP), dislipidemias, anemias e outros fatores como idade, gênero, tabagismo, etilismo e sobrepeso, pode favorecer o seu desenvolvimento riores, apresentam-se como ferida única e dolorosa com aparência irregular e é superficial. Estas lesões apresentam rápido crescimento e alterações da hipertensão arterial dificultando o controle da dor. A UHM é mais prevalente em pacientes do gênero feminino com idades entre 55 e 65 anos, porém é subdiagnosticada o que pode corroborar inclusive para baixa disponibilidade de pesquisas sobre a lesão ${ }^{(7,8,10)}$.

O objetivo do estudo foi discutir e investigar as formas de diagnóstico e tratamento do paciente com Úlcera Hipertensiva de Martorell (UHM).

MÉTODO

Trata-se de um estudo descritivo e qualitativo, realizado por meio de uma revisão integrativa de literatura sobre a abordagem da temática DA Úlcera Hipertensiva de Martorell, buscando responder à seguinte questão norteadora: "Quais os métodos de diagnóstico e os tratamentos existentes para a Úlcera Hipertensiva de Martorell (UHM)?".

Para o levantamento bibliográfico, foram realizadas estratégias de busca utilizando os Descritores e suas combinações na língua portuguesa e inglesa: "Ulcera de Perna" e "Ulcera cutânea". A seleção dos estudos obedeceu aos seguintes critérios de inclusão: publicações indexadas ou catalogadas eletronicamente disponíveis na íntegra nas bases de dados Pubmed e BVS, no período de 2016 a 2021 que abordavam a temática proposta. Foram encontrados 566 trabalhos, excluídos trabalhos repetidos, e após a leitura dos resumos foram selecionados 44 (quarenta e quatro) artigos, que possuíam relevância a temática abordada. Na pesquisa de literatura não retornam artigos da revista Nursing. Após leitura dos trabalhos na integra, foram excluídos vinte e cinco trabalhos pelo não enquadramento da abordagem temática. A estratégia de busca encontra-se esquematizada na figura 1 . 


\begin{tabular}{|c|c|c|}
\hline \multicolumn{3}{|r|}{ Quadro 1 - Síntese dos artigos selecionados } \\
\hline $\begin{array}{l}\text { Autores e Ano de } \\
\text { Publicação }\end{array}$ & Título & Principais resultados \\
\hline $\begin{array}{l}\text { Chicote-Álvarez et } \\
\text { al. }(2019)^{(11)}\end{array}$ & $\begin{array}{l}\text { Úlceras hipertensivas de } \\
\text { Martorell. Una compli- } \\
\text { cación infrecuente de la } \\
\text { hipertensión artéria }\end{array}$ & $\begin{array}{c}\text { Estudo de caso de paciente masculino, } 51 \text { anos, hipertensão descontrolada, DM } 2 \text { e reabilitação } \\
\text { após sofrer um acidente vascular cerebral isquêmico após embolia e apresenta úlcera de Martorell. } \\
\text { Foi verificado que o tratamento mais rápido é a cirurgia, para controle adequado da dor, com desbri- } \\
\text { damento do tecido necrótico. }\end{array}$ \\
\hline
\end{tabular}

A Novel Association of Estudo de caso de tratamento cirúrgico de uma úlcera de Martorell em uma mulher de 69 anos com Malphrus (2019) $\quad$ Martorell Ulcer With Liddle síndrome de Liddle. É o primeiro caso relatado na literatura desta úlcera. É necessário mais estudos Syndrome para facilitar o diagnóstico e tratamento.

\begin{tabular}{|c|c|c|}
\hline Munoz et al. (2019(7) & $\begin{array}{l}\text { Tratamiento de la úlcera } \\
\text { isquémica hipertensiva de } \\
\text { Martorell con microinjer- } \\
\text { tos autólogos en sello }\end{array}$ & $\begin{array}{l}\text { Nos } 3 \text { casos de UIHM apresentados, a terapia de microenxerto autólogo (MAS) permitiu períodos de } \\
\text { cicatrização inferiores a } 2 \text { meses, com controle da dor associada. É uma opção terapêutica segura e } \\
\text { eficaz, com simples aplicação ambulatorial e com baixa morbidade para o paciente. }\end{array}$ \\
\hline Kolius et al. $(2018)^{13)}$ & $\begin{array}{l}\text { Comparison of pyoderma } \\
\text { gangrenosum and Marto- } \\
\text { rell hypertensive ischaemic } \\
\text { leg ulcer in a Swiss cohort }\end{array}$ & $\begin{array}{c}\text { O pioderma gangrenoso (PG) é uma dermatose neutrofilica rara, apresenta ulcerações cutâneas } \\
\text { dolorosas e estéreis, de etiologia desconhecida. Estudos prospectivos maiores ou registros internacio- } \\
\text { nais sobre essas doenças cutâneas ulcerativas raras são necessários para confirmar esses achados e } \\
\text { facilitar o diagnóstico e tratamento. }\end{array}$ \\
\hline $\begin{array}{l}\text { Wirsing et al. } \\
\left(2019^{(14)}\right.\end{array}$ & $\begin{array}{l}\text { Martorell's Ulcer Succes- } \\
\text { sfully Treated by Wireless } \\
\text { Microcurrent Stimulation } \\
\text { Technology }\end{array}$ & $\begin{array}{l}\text { Estudo de caso sobre o uso de estimulação de microcorrente sem fio, para a cura da Úlcera de Mar- } \\
\text { torell. A estimulação de microcorrente sem fio, reduziu o tamanho de uma grande úlcera de Martorell } \\
\text { por } 90 \% \text { em } 8 \text { semanas. A comparação com os métodos tradicionais de cicatrização de úlceras com } \\
\text { a estimulação de microcorrente sem fio demonstrou eficacia do uso desta tecnologia. }\end{array}$ \\
\hline Serpa et al. $(2018)^{(15)}$ & $\begin{array}{c}\text { A Challenging Diagnosis } \\
\text { of Leg Ulcer }\end{array}$ & $\begin{array}{l}\text { A úlcera hipertensiva de Martorell é amplamente ignorado e subdiagnosticado. Paciente afro-ame- } \\
\text { ricana com hipertensão de longa duração que apresentou uma úlcera de perna muito dolorosa. } 0 \\
\text { diagnóstico foi complicado pela coexistência de causas mais comuns de úlcera mas foi possivel pela } \\
\text { biopsia.0 tratamento padrão é o controle adequado da pressão arterial. }\end{array}$ \\
\hline Hafner $(2016)^{(9)}$ & $\begin{array}{l}\text { Calciphylaxis and Marto- } \\
\text { rell Hypertensive Ischemic } \\
\text { Leg Ulcer: Same Pattern - } \\
\text { One Pathophysiology }\end{array}$ & $\begin{array}{l}\text { Estudo apresenta a comparação da: calcifilaxia (padrão distal); calcifilaxia; úlcera de perna isquêmica } \\
\text { hipertensiva de Martorell e calcifilaxia com função renal e paratireóide normais.Os quatro diagnós- } \\
\text { ticos são pouco conhecidos na área médica na maioria dos países, fato que dificulta o diagnostico } \\
\text { diferencial e o tratamento dessas doenças. }\end{array}$ \\
\hline
\end{tabular}

Fonte: Autores, 2021

Conforme esquematizado na Figura 1 , foram selecionados dezenove artigos que realizavam a abordagem da Ulcera Hipertensiva de Martorell (UHM).

\section{RESULTADOS}

Foram selecionados para este estudo 07 (sete) artigos correspondentes aos critérios de inclusão. Dentre eles, cinco $(71,4 \%)$ retratam estudo de casos, descrevendo a dificuldade do diagnostico diferencial e tratamento, e concluem a existência de poucos estudos sobre a Ulcera de Martorell; e apenas dois $(28,6 \%)$ estudos realizam discussão da Ulcera de Martorell através da comparação com outras doenças, enfatizando a dificuldade de diagnostico para o tratamento adequado. Os principais resultados dos estudos selecionados podem ser observados no Quadro 1.

Embora a etiologia da UHM não esteja totalmente esclarecida, vários fatores estão relacionados ao seu desenvolvimento. Entre os motivos estão a resistência vascular aumentada na região da ferida responsável por infarto nas arteríolas e necrose tecidual, resposta arteriolar atípica a componente vasoativo, alterações na inervação simpática, hipertonia arteriolar constante e trauma que interfere na circulação terminal A fisiopatologia da ferida está relacionada a alterações arterioscleróticas devido ao mau controle da hipertensão arterial que pode levar ao desenvolvimento de hialinose responsável pelo aumento da resistência vascular local e diminuição da pressão de perfusão no tegumento ${ }^{(7,11)}$.

A prevalência da Úlcera hipertensiva de Martorell (UHM) é em indivíduos com mais de 60 anos e com hipertensão arterial sistêmica de longa duração sem controle adequado da doença. Existe a associação com patologias de base como obesidade, insuficiência venosa crônica e doença arterial periférica. Além disso, cerca de $60 \%$ dos pacientes com UHM são diabéticos (tipo 2) e apresentam pressão em tornozelo $\geq 60 \mathrm{mmHg}^{(6,7,9)}$.

A principal forma de diagnóstico da UHM é fundamentalmente clínica, por exclusão de outras causas. O diagnósti- 
co definitivo é determinado pela biópsia através da análise histopatológica. A amostra deve ser colhida através de tecidos saudáveis e afetados e ter comprimento suficiente e profundidade, medindo $6 \mathrm{~cm}$ de comprimento, $6 \mathrm{~mm}$ de largura e 6-40 mm de largura profundidade e se estender até a fáscia da perna ou em outros locais nas profundezas do subcutâneo. Os achados histopatológicos encontrados são lesões obstrutivas em arteríolas subcutâneas devido ao espessamento de endotélio, da camada média e hialinose arterio$\operatorname{lar}^{(7,9,12-15)}$.

A UHM apresenta-se inicialmente com o infarto da pele de uma área lívida e dolorida, geralmente caracterizada por livedo racemosa, que rapidamente se torna necrótico e margem com estenose subcutânea relacionada a arteriolosclerose, acompanhada de calcinose medial. A ferida tem aspecto arredondado, superficial, geralmente pequena com margens necróticas e cianóticas, associado a eritema cutâneo perilesional. Os pulsos distais estão presentes e há ausência de edema e varizes local ${ }^{(9,12,15-19)}$.

Os pacientes com UHM, em 90\% dos casos, apresentam uma lesão mais comumente na região maleolar lateral (anterolateral ou supramaleolar), com evolução progressiva de necrose cutânea e extremamente dolorosa, com uma predisposição marcada para a parte inferior laterodorsal da perna ou acima do tendão de Aquiles. Pode surgir espontaneamente $(44,4 \%$ dos casos) ou secundária à trauma local $(55,6 \%$ dos casos $)^{(6,9,12,14,15,18,19)}$.

Entre os sinais e sintomas relatados pelos pacientes com UHM, destaca-se a dor. A graduação da dor é intensa e desproporcional à área da ferida. A causa está relacionada a estimulação das terminações nervosas que geram a dor e a instabilidade local de eletrólitos e do $\mathrm{pH}$, além da presença de tecido necrótico e/ou inviável. O controle da dor do doente é parte fundamental do
A UHM apresentase inicialmente com o infarto da pele de uma área lívida e dolorida, geralmente caracterizada por livedo racemosa, que rapidamente se torna necrótico

e margem com estenose subcutânea relacionada a arteriolosclerose, acompanhada de calcinose medial. plano terapêutico e na maioria das vezes é necessário a prescrição de analgésicos potentes à base de codeína, tramadol e pregabalina, além de vasodilatadores periféricos como o cilostazol, respeitando as contraindicações que houver. Os tratamentos cirúrgicos como desbridamento e enxerto podem ser necessários para auxiliar na cicatrização da ferida $a^{(5,6,9,11,12,18,19)}$.

A assistência do paciente com UHM preferencialmente deve acontecer por equipe multidisciplinar de profissionais de saúde e com abordagem transdisciplinar formada por enfermeiros, fisioterapeutas, médicos, nutricionistas, dentre outros ${ }^{(9)}$.

Alguns autores sugerem o controle dos níveis da pressão arterial sistêmica de acordo com os recomendados pelas Sociedades Brasileira de Cardiologia com o uso de medicamentos anti-hipertensivos como tratamento de primeira linha de pacientes com úlcera Hipertensiva de Martorell, para contribui com o processo de cicatrização da lesão. Estudos sugerem ainda a combinação com agentes anti-hipertensivos seletivos, como bloqueadores dos canais de cálcio e enzima conversora de angiotensina inibidores ou com outros bloqueadores A1 seletivos anti-hipertensivos que reduzem a vasoconstrição local, incluindo alta seletividade bloqueadores como bisopro ou nebivolol. Devido à dor extrema causada pela ferida, mesmo com o tratamento anti-hipertensivo adequado, a maioria dos pacientes desenvolvem picos de hipertensão arterial que pode exigir adequações nas medicações ${ }^{(7,9,20,21)}$.

Alguns estudos, recomendam o desbridamento precoce e enxerto de pele para melhorar cicatrização da ferida e alívio da dor. A maioria dos pacientes requerem um ajuste de resistência antibioticoterapia sistêmica neste tratamento cirúrgico agudo. Quando o procedimento de enxerto de pele é bem-sucedido, normalmente, a dor severa da ferida desaparece com uma ra- 
pidez surpreendente a um nível suportável em aproximadamente 1-2 dias.

Geralmente é possível realizar cura da ferida remanescente ao longo de semanas e meses de tratamento conservador e com boa qualidade de vida. Cerca de $30-40 \%$ dos pacientes requerem 2 e às vezes até 3 enxertos de pele para interromper o surgimento de necrose e favorecer o processo de regeneração tecidual ${ }^{(9)}$.

Os enfermeiros desempenham uma função fundamental na avaliação e gestão de pacientes com UHM, fornecendo uma variedade de cuidados como curativos, orientações sobre os medicamentos prescritos e coordenar o envolvimento de outros profissionais de saúde. Para os curativos recomendam-se coberturas interativas capazes de favorecer a regeneração do tecido lesado com manutenção ideais de umidade, temperatura e neoangiogênese $\mathrm{e}^{(6,11)}$.

\section{DISCUSSÃo}

A Úlcera Hipertensiva de Martorell (UHM) é uma síndrome rara, amplamente ignorada e subdiagnosticada. Nos casos de confirmação de Úlcera Hipertensiva de Martorell, estes pacientes possuem hipertensão arterial e diabetes e apresentam a úlcera típica é na perna inferior, é extremamente dolorosa e se apresenta em pacientes do gênero feminino com mais de 60 anos de idade, com fatores de risco cardiovascular, principalmente hipertensão arterial sistêmica grave, além de dor excruciante desproporcional a área da ulceração ${ }^{(15,16,18-20)}$.

É um desafio caracterizar a UHM, visto a identificação de poucos estudos sobre o assunto ${ }^{(13,18,19)}$.

Entre os desafios encontrados, está o diagnóstico diferencial dentre as feridas crônicas. As apresentações clínicas, a etiologia subjacente e as manifestações patológicas das úlceras são as principais pistas para o diagnóstico. Estudos demonstraram que $50 \%$ de 31 casos de pacientes com Úlcera Hipertensiva de Martorell foram diagnosticados erroneamente como aspyoderma gangrenoso e $20 \%$ como vasculite necrosante $^{(17,19,20)}$.

O tratamento objetiva o controle da HAS e o controle de da dor com analgésicos orais ou simpatectomia lombar (para bloquear o vasoespasmo e reduzir a resistência vascular) podem ser considerados. Os narcoticanalgésicos devem ser administrados em conjunto com pregabalina ou antidepressivos tricíclicos, devido dor neuropática, que é uma queixa comum apresentada entre os pacientes acometidos com a $\mathrm{UHM}^{(19-21)}$

\section{CONCLUSÃO}

Estudos sobre UHM são escassos o que pode corroborar para o subdiagnóstico da doença. Foi evidenciado a dificuldade no diagnóstico e tratamento dos pacientes devido à falta de conhecimento dos profissionais sobre essa doença. O diagnóstico da UHM é clínico e confirmada por análise histopatológica através da biopsia da ferida. O tratamento inclui controle da HAS, medicamentos, curativos, e procedimentos cirúrgicos e controle da dor com a prescrição de medicações analgésicas para o conforto do paciente. O tratamento é complexo e desafia profissionais de saúde durante a assistência do paciente. O tratamento multidisciplinar pode otimizar a cicatrização da ferida, diminuindo o tempo do tratamento e, portanto, o sofrimento do doente. É necessário discutir os casos clínicos e realizar novos estudos para ampliar as possibilidades de diagnóstico e tratamento precoce, que permitam diminuir novas ocorrências e tempo de tratamento e cicatrização das UHM.

\section{Referências}

1. Cortez DN, Moraes JT, Ferreira IR, Silva EL, Lanza FM. Custos do tratamento de lesões cutâneas na Atenção Primária à Saúde. Estima. 2019;17:1-7.

2. Borges EL, Nascimento Filho HM, Pires Junior JF. Prevalência de lesões crônicas de município da Zona da Mata Mineira (Brasil). Rev Min Enferm. 2018;22:1-7.

3. Martínez-Santos AE, Bouza ET, Cartelle JAP. Conocimiento enfermero sobre prevención, diagnóstico y cuidados de las úlceras de extremidad inferior en un área sanitária. Gerokomos. 2019;30(1):34-41.

4. Barber GA, Weller CD, Gibson SJ. Effects and associations of nutrition in patients with venous leg ulcers: a systematic review. J Adv Nurs. 2017;74(4):774-87.

5. Loos M, Coringrato M, Liliana O. Úlcera hipertensiva de Martorell. Dermatol Argent. 2017;23(2):58-65.

6. Calle JFJ, Marín SGY, Carvajal CAS, Mena VPC. Ulcera de Martorell: diagnóstico. Recimundo. 2020;4(1):54-62.

7. Munoz SG, Montero EC, Dobao PC. Tratamiento de la úlcera isquémica hipertensiva de Martorell com microinjertos autólogos ensello. Rev Actas Dermosifiliogr. 2019;110(8):689-90.

8. Malaspina PSB, Gorosito SE, Petovello F, Márquez M, Trila C, Miravalle D. Diagnóstico y tratamiento de la úlcera de Martorell. Rev Flebología. 2016;42(3):37-40.

9. Hafner J. Calciphylaxis and Martorell Hypertensive Ischemic Leg Ulcer: same pattern - One pathophysiology. Dermatology 2016;232(5):523-33.

10. Bachero JG, Baldrich YG, Palasí CR. Caso de una úlcera hipertensiva de Martorell: importancia de una buenaanamnesis y diagnóstico para suresolución. Enferm Dermatol. 2017;11(30):57-63

11. Chicote-Álvareza E, Santos-Méndez N, Lizama-Gómez NG, Ortiz-Flores $F$. Úlceras hipertensivas de Martorell: una complicación infrecuente de la hipertensión arterial. Soc Esp Med Rural Gen. 2019:45(8):49-50. 


\section{Referências}

12. Malphrus E, Couch K, Chao JW. A Novel Association of Martorell ulcer with liddle syndrome. Adv Skin Wound Care. 2019;32(11):1-2.

13. Kolius AGA, Hafner J, Luder C, Guenova E, Kerl K, Kemp W, et al. Comparison of pyoderma gangrenosum and Martorell hypertensive ischaemic leg ulcer in a Swiss cohort. Br J Dermatol. 2018;178(2):125-6.

14. Wirsing PG, Konstantakaki M, Poulas AK. Martorell's Ulcer Successfully treated by wireless microcurrent stimulation technology. Adv Skin Wound Care. 2019;32(2):81-4.

15 Serpa MJ, Franco S, Repolho D, Araújo I, Mateus S, Baptista AM, et al. A challenging diagnosis of leg ulcer. Eur J Case Rep Intern Med. 2018 24;5(10):1-4.

16. Pinto APFL, Silva-Júnior NA, Osório CT, Rivera LM, Carneiro S, Ramos-e-Silva M, et al. Martorell's ulcer: diagnostic and therapeutic challenge. Case Rep Dermatol. 2015;7(2):199-206.

17. Pontes AAN, Macieira NFZ, Sousa RJ. Úlcera de Martorell: análise epi- demiológica e clínica em pacientes portadores de diabetes mellitus tipo 2. Rev Saúde Ciênc. 2017;6(2):5-11.

18. Malhi HK, Didan A, Ponosh S, Kumarasinghe SP. Painful leg ulceration in a poorly controlled hypertensive patient: a case report of Martorell ulcer. Case Rep Dermatol. 2017;9(1):95-102.

19. Estrela FM, Soares VA, Moreira BSG, Gomes NP, Santana LC, Lima CCOJ. Úlcera hipertensiva: uso da cobertura de espuma de poliuretano com ibuprofeno. Rev Pelle Sana. 2016;3:54-61.

20. Fiçıcıoðlu S, Can N, Tutu囚 B. A case of chronic ulcer due to subcutaneous arteriolosclerosis in an obese patient mimickingpyoderma gangrenosum. Dermatol Reports. 2018;10(1):7445.

21. Malhi HK, Didan A, Ponosh S, Kumarasinghe SP. Painful leg ulceration in a poorly controlled hypertensive patient: a case report of Martorell ulcer. Case Rep Dermatol. 2017;9(1):95-102. 